\title{
Hypoxia-inducible factors: a central link between inflammation and cancer
}

\author{
Daniel Triner ${ }^{1}$ and Yatrik M. Shah ${ }^{1,2}$ \\ 'Department of Molecular and Integrative Physiology and ${ }^{2}$ Department of Internal Medicine, Division of Gastroenterology, University of Michigan Medical School, Ann Arbor, Michigan, USA.
}

\begin{abstract}
The tumor immune response is in a dynamic balance between antitumor mechanisms, which serve to decrease cancer growth, and the protumor inflammatory response, which increases immune tolerance, cell survival, and proliferation. Hypoxia and expression of HIF-1 $\alpha$ and HIF- $2 \alpha$ are characteristic features of all solid tumors. HIF signaling serves as a major adaptive mechanism in tumor growth in a hypoxic microenvironment. HIFs represent a critical signaling node in the switch to protumorigenic inflammatory responses through recruitment of protumor immune cells and altered immune cell effector functions to suppress antitumor immune responses and promote tumor growth through direct growth-promoting cytokine production, angiogenesis, and ROS production. Modulating HIF function will be an important mechanism to dampen the tumor-promoting inflammatory response and inhibit cancer growth.
\end{abstract}

\section{Introduction}

The tumor microenvironment is similar to an inflammatory focus, as it consists of a complex milieu of both innate and adaptive immune cells (1). Hypoxia is a characteristic feature of both tumors and inflammatory foci. Increased metabolic demand from rapid cell turnover, immune cell infiltration, and vascular disruption cause local $\mathrm{O}_{2}$ tension to decline. The decreased $\mathrm{O}_{2}$ tension of tumors or inflamed tissue promotes activation of HIFs. HIFs are basic helix-loop-helix-Per-ARNT-Sim-containing (bHLH-PAScontaining) transcription factors consisting of a heterodimer of an oxygen-sensitive $\alpha$ subunit (HIF-1 $\alpha$, HIF-2 $\alpha$, and HIF-3) and a constitutively expressed $\beta$ subunit (ARNT) (2). HIF-1 $\alpha$ is ubiquitously expressed, whereas HIF- $2 \alpha$ and HIF-3 $\alpha$ expression is largely tissue restricted (3-5). HIF- $\alpha$ subunits are regulated by $\mathrm{O}_{2}$-dependent posttranslational hydroxylation of two specific proline residues by prolyl hydroxylase domain-containing (PHD-containing) enzymes. In normoxia, HIF hydroxylation leads to association with the von Hippel-Lindau (VHL) tumor suppressor/E3 ubiquitin ligase complex, ubiquitin conjugation, and 26S proteasomal degradation. As $\mathrm{O}_{2}$ homeostasis is disrupted and $\mathrm{O}_{2}$ concentration declines under inflammatory conditions or in tumors, HIFs are stabilized, dimerize with ARNT, and translocate to the nucleus to regulate transcription by binding to hypoxia response elements (HREs) in promoters of target genes (Figure 1A).

In addition to $\mathrm{O}_{2}$-dependent regulation, inflammation and direct HIF regulation are intimately linked. NF- $\kappa \mathrm{B}$, a master transcription factor in the inflammatory response, is a direct transcriptional regulator of Hifla. In response to NF- $\kappa \mathrm{B}$-activating stimuli such as bacterial lipopolysaccharide (LPS), NF- $\kappa \mathrm{B}$ directly increases Hifla mRNA in macrophages (6). LPS-induced NF- $\kappa B$ can also enhance HIF- $1 \alpha$ protein stability by increasing intracellular ferritin, which sequesters the labile iron pool and leads to

Conflict of interest: The authors have declared that no conflict of interest exists. Reference information: / Clin Invest. 2016;126(10):3689-3698. doi:10.1172/JCI84430. decreased PHD activity (7). Independently of NF-кB, several cytokines and intermediate metabolites such as succinate can lead to HIF activation $(8,9)$. In macrophages, IL-4 and IL-13 selectively induce Hif $2 a$ mRNA (10). Several studies have demonstrated that cytokine-induced ROS and specifically mitochondrial ROS directly activate HIF (11-13), and recently it was shown that mitochondrial membrane potential increases mitochondrial ROS to modulate HIF activation (14) (Figure 1B).

HIFs are critical drivers of cancer and regulate a wide variety of cellular processes including metabolism, cell cycle progression, angiogenesis, invasion/metastasis, and chemoresistance (15). HIF- $1 \alpha$ and HIF- $2 \alpha$ are highly expressed in a wide variety of solid tumors, including those of the colon, breast, lung, and pancreas (16). Although HIF-1 $\alpha$ and HIF-2 $\alpha$ have several overlapping functions, distinct target genes and functions for HIF- $1 \alpha$ and HIF- $2 \alpha$ are well characterized (17) and highlighted in several tumor models (18). In renal cell carcinoma (RCC) that is deficient in $V H L$, which stabilizes HIF- $1 \alpha$ and HIF- $2 \alpha$ in normoxic conditions, HIF- $1 \alpha$ has an antitumor role and decreases tumor growth by increasing expression of proapoptotic genes (19). HIF-2 $\alpha$ is essential for RCC tumor growth and promotes tumor cell proliferation through augmented c-Myc activity (20). It is well known that hypoxia and HIF signaling play an important role in inflammatory responses and regulating the immune environment (21); however, the intersection of hypoxia, inflammation, and cancer is not well understood. In this review we will discuss the role of hypoxia in modulating the protumor inflammatory response or antitumor immune response in cancer.

\section{Inflammation and cancer}

It is widely recognized that inflammation plays an essential role in tumorigenesis and tumor progression. This paradigm dates back to Rudolf Virchow, a 19th century pathologist whose observation of leukocytes in tumors led him to hypothesize that tumors originate in sites of chronic inflammation. The discovery of the first oncogene $v$-src in Rous sarcoma virus over a century ago led to decades 
A

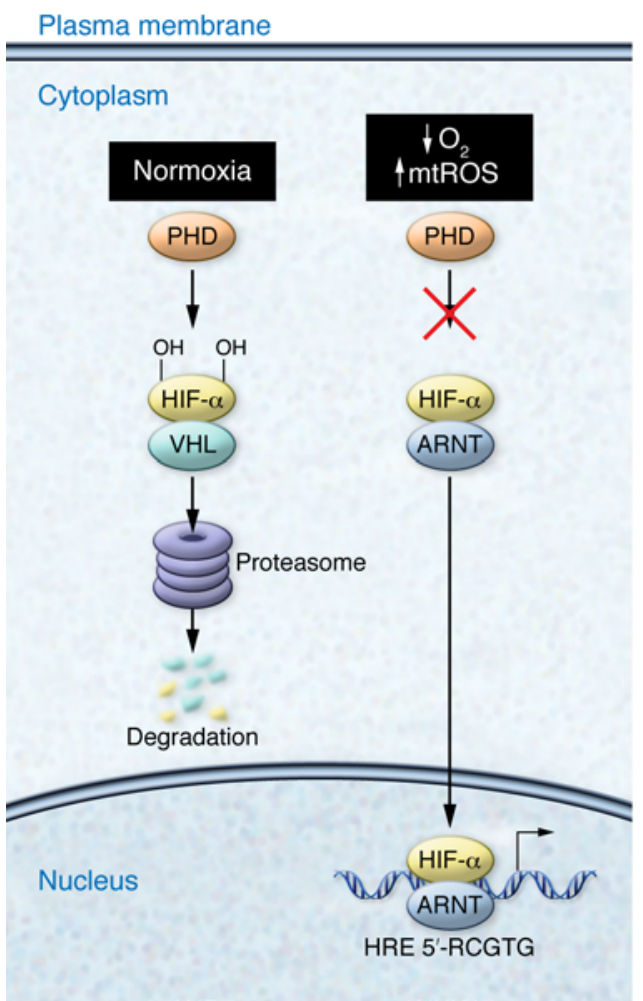

B

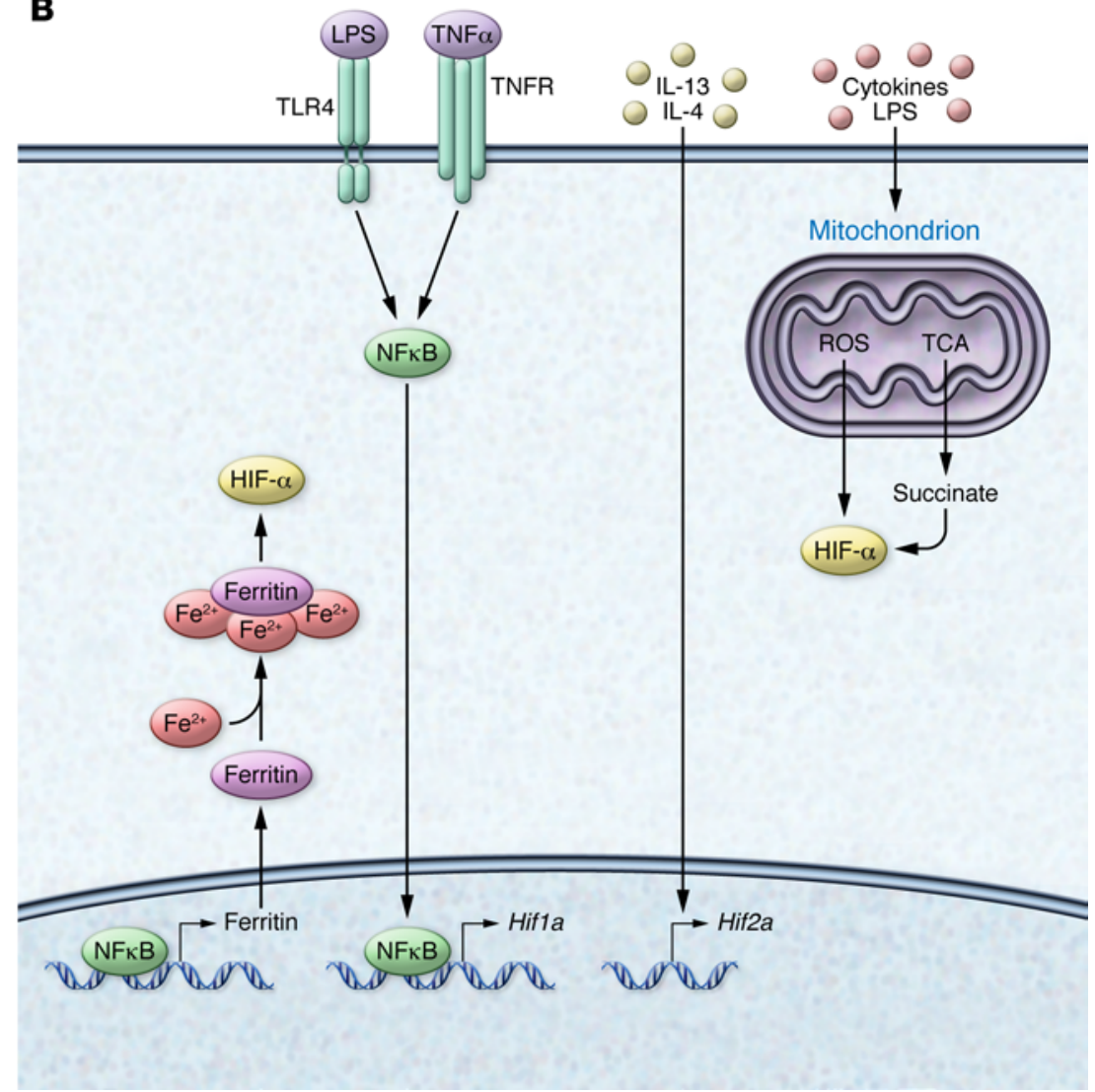

Figure 1. Activation of HIFs by hypoxia and inflammation. (A) HIF- $\alpha$ subunits are regulated by posttranslational hydroxylation of two specific proline residues by PHD enzymes in normoxic conditions. This leads to VHL-mediated proteasomal degradation. As $\mathrm{O}_{2}$ concentration drops or mitochondrial ROS production increases HIF- $\alpha$ subunits are stabilized, dimerize with ARNT, and translocate to the nucleus and bind to HRE sequences in target genes. (B) HIFs are activated by inflammation through cytokine-induced Hif2 $\alpha$ expression, NF- $\kappa B$-mediated transcription of Hif1 $\alpha$, NF- $\kappa B-d e p e n d e n t$ ferritin iron sequestration, cytokine-mediated mitochondrial ROS production, and the TCA metabolite succinate.

of in-depth cancer genetic analysis and the discovery of numerous oncogenes and tumor suppressors; however, studies of tumorassociated inflammation lagged behind. A renaissance over the last 20 years has uncovered a critical role for inflammation in the pathogenesis and progression of nearly all solid tumors, and this knowledge has greatly changed the approach to current cancer treatments. The advent of immune-modulating therapies, such as immune checkpoint blockers for the treatment of advanced cancers, underscores the importance of understanding the specific function of inflammatory cells in cancer and is an exciting avenue for the discovery of novel therapeutic targets (22).

Chronic inflammation-associated cancers. As Virchow postulated, several chronic inflammatory diseases predispose the development of cancer. For example, Helicobacter pylori, a gramnegative bacterium, infects nearly $50 \%$ of the world's population and is the major causative agent of chronic gastritis (23). Chronic gastritis associated with $H$. pylori is asymptomatic in the majority of infected individuals; however, this chronic gastritis represents a significant risk factor for the development of gastric cancer, which occurs in $1 \%-3 \%$ of $H$.pylori infections and is the third leading cancer type worldwide (23). Similarly, chronic viral infections predispose to the development of cancer. Hepatitis B virus (HBV) and hepatitis $\mathrm{C}$ virus ( $\mathrm{HCV})$ infections are key risk factors for development of hepatocellular carcinoma (HCC). For example, HCV infects more than 100 million people worldwide, and HCC due to chronic hepatitis induced by HCV infection occurs in approximately $1 \%-5 \%$ of infected individuals (24). One of the best-studied cancers from a genetic and inflammatory perspective has been colon cancer. Chronic intestinal inflammation associated with inflammatory bowel disease (IBD), including Crohn's disease and ulcerative colitis, represents a significant risk factor for the development of colon cancer, termed colitis-associated colon cancer (CAC). More than 1 million Americans suffer from IBD and $12 \%-20 \%$ of patients will develop CAC within 30 years of developing IBD (25). The vast majority of colon cancers develop sporadically and proceed through a step-wise process from adenoma to invasive carcinoma (26). This process includes the acquisition of sequential mutations leading to the loss of adenomatous-polyposis coli (APC) tumor suppressor, activation of the oncogene KRAS, and loss of the TP53 tumor suppressor (27). Interestingly, in colon cancers preceded by chronic inflammation due to IBD, the kinetics of the genetic alterations are different, as TP53 is lost early in disease progression and APC mutations are not as frequently observed compared to sporadic colon cancer $(28,29)$. These data suggest that inflammation may induce novel mechanisms to drive cellular proliferation and survival, resulting in tumorigenesis. 
Tumor-derived inflammation. All solid tumors elicit an inflammatory response that is critical in the tumor microenvironment. The tumor-derived inflammatory response is essential for the recruitment of immune cells, tumor cell proliferation, survival, and angiogenesis (30). Tumors initiate these responses through several mechanisms including transcriptional regulation of inflammatory genes by proto-oncogenes $(31,32)$. It was recently demonstrated that defects in epithelial permeability elicited an inflammatory response through a microbiota-mediated mechanism in colon cancer (33). Below, we illustrate the importance of hypoxia in mediating the tumor-derived inflammatory response.

Pro- and antitumor immune responses. In tumors, there is a mix of both innate and adaptive immune cells with anti- and protumor functions (1). Precise identification of immune cells found in tumor biopsies can serve as prognostic markers for clinical outcomes. Adaptive immune responses have been correlated with positive prognosis. Several distinct $\mathrm{T}$ cell subsets reside within the tumor microenvironment, including cytotoxic antitumor $\mathrm{CD}^{+}$ $\mathrm{T}$ cells and subsets of $\mathrm{CD}^{+} \mathrm{T}$ cells with both pro- and antitumor functions. In colon cancer, decreased expression of CD8 as well as the cytotoxic T cell markers granzyme B and CD45RO all correlated with disease recurrence. Furthermore, patients with high intratumoral expression of the $\mathrm{T}$ cell marker CD3 had improved disease-free survival compared to patients with low levels of CD3 (34). Recent meta-analysis studies have mapped the prognostic impact of 22 immune cell types on recurrence-free survival by analyzing expression signatures across more than 5,000 tumor samples (35). In general, these studies found a positive correlation between $\mathrm{T}$ cells and recurrence-free survival and a negative correlation for several myeloid cell types such as neutrophils and macrophages (35). In ovarian cancer, an increased $\mathrm{CD} 8^{+} / \mathrm{CD} 4^{+} \mathrm{T}$ cell ratio was associated with improved survival, whereas increased $\mathrm{CD}^{+} \mathrm{T}$ cells portended poor survival (36). Expression profiles associated with antitumor T cell cytokines (IFN- $\gamma$ ) and effector molecules (granulysin and granzyme B) are also independent prognosticators of decreased early metastasis in colon cancer (37). These associations, however, do not highlight the complexity of the tumor immune environment. Many specific cell types have been associated with better or worse prognosis, and the plasticity of immune cells can confer both pro- and antitumor functions, which we will review here in depth.

\section{Hypoxia and epithelial-elicited tumor inflammatory response}

Historically it was believed that the major function of epithelial surfaces, such as the skin or the intestinal epithelium, was to serve as a physical barrier separating the external environment from the underlying immune cells. It is now evident that epithelial surfaces play an active role in innate immunity and shape the underlying immune environment and inflammatory response $(38,39)$. This is also the case in epithelial-derived cancers. Colon tumor cell secretion of the C-C family chemokine, CCL2, was essential for tumorigenesis through recruitment and activation of protumorigenic myeloid cells (40). Pentraxin 3 (PTX3) is a tumor suppressor that activates complement-mediated antitumor immunity and is epigenetically silenced due to methylation in colon tumors (41). Recent studies in colon cancer have further highlighted the contribution of epithelial and stromal gene expression in patientderived colon cancer xenograft models (42). In these studies, the epithelial and stromal gene expression was readily delineated by analyzing tumor expression of human or mouse transcripts. Analysis of genes with a greater than $50 \%$ difference in expression between the epithelium and the stroma showed that several cytokines and chemokines are directly expressed by the tumor epithelium (42). These results demonstrate that tumor epithelial cells play an active role in regulating the inflammatory response, which may impact tumorigenesis.

Several lines of evidence suggest that intratumoral hypoxia and HIFs play an essential role in sculpting the tumor immune environment in epithelial-derived tumors. For example, in a Krasdriven mouse model of non-small cell lung cancer (NSCLC), loss of HIF- $2 \alpha$ increased tumor burden and tumor cell proliferation; however, loss of HIF-1 $\alpha$ had no effect on tumorigenesis (43). Tumors lacking HIF- $2 \alpha$ also displayed increased infiltration of CD $45^{+}$immune cells, specifically Gr- $1^{+}$granulocytic cells, suggesting that HIF-2 $\alpha$ repression of granulocytic cell infiltration is in part responsible for its antitumor effects (43). Hypoxia and HIF stabilization is also a key feature of pancreatic ductal adenocarcinoma (PDAC) (16). PDAC development occurs in a step-wise manner and is preceded by precursor lesions termed pancreatic intraepithelial neoplasias (PanINs) (44). In contrast with studies showing decreased PanIN progression following pancreatic-specific disruption of HIF- $2 \alpha$ (45), deletion of HIF- $1 \alpha$ in a murine model of Krasinitiated PDAC significantly enhanced PanIN progression and increased tumor cell proliferation (46). Loss of HIF- $1 \alpha$ correlated with increased pancreatic B cell infiltration and antibody-mediated depletion of $\mathrm{B}$ cells reversed the increased PanIN progression (46). Hypoxic inflammation is also important in colon tumorigenesis, and both HIF- $1 \alpha$ and HIF- $2 \alpha$ are overexpressed in colon tumors (16). Using the $A p c^{m i n /+}$ model of intestinal tumorigenesis, intestine-specific disruption of the tumor suppressor $V h l$ significantly increased colon tumorigenesis and adenoma-to-carcinoma progression (47). The increase in colon tumorigenesis was HIF- $2 \alpha$ dependent, as double disruption of $V h l$ as well as Hif $2 a$ ameliorated the effect (47). HIF-2 $\alpha$-mediated inflammatory responses are essential in colon tumorigenesis. Epithelial HIF- $2 \alpha$ regulates expression of the proinflammatory meditator TNF- $\alpha$ (Tnfa) (48). TNF- $\alpha$ has a crucial role in the progression of cancer, and inhibiting TNF- $\alpha$ decreases growth in several mouse models of cancer $(49,50)$. HIF-2 $\alpha$-induced inflammation was found to be critical to tumor progression, as treatment with the antiinflammatory drug nimesulide significantly reduced HIF- $2 \alpha$-driven colon tumorigenesis. (48). Intestine-specific overexpression of HIF-1 $\alpha$ does not enhance tumorigenesis in colon cancer (51). These studies suggest that tumor HIFs have the potential to modulate tumor-associated inflammation to regulate tumor growth and progression.

\section{Hypoxia and immune cell recruitment}

Tumors are highly infiltrated by cells of both the innate and adaptive immune systems. This infiltration is partially mediated by tumor-derived secretion of a host of cytokines and chemokines (Figure 2A). HIF-induced secretion of tumor-derived factors can modulate immune cell recruitment to aid in tumor growth, which we will review in detail by immune cell subtype (Table 1). 
A

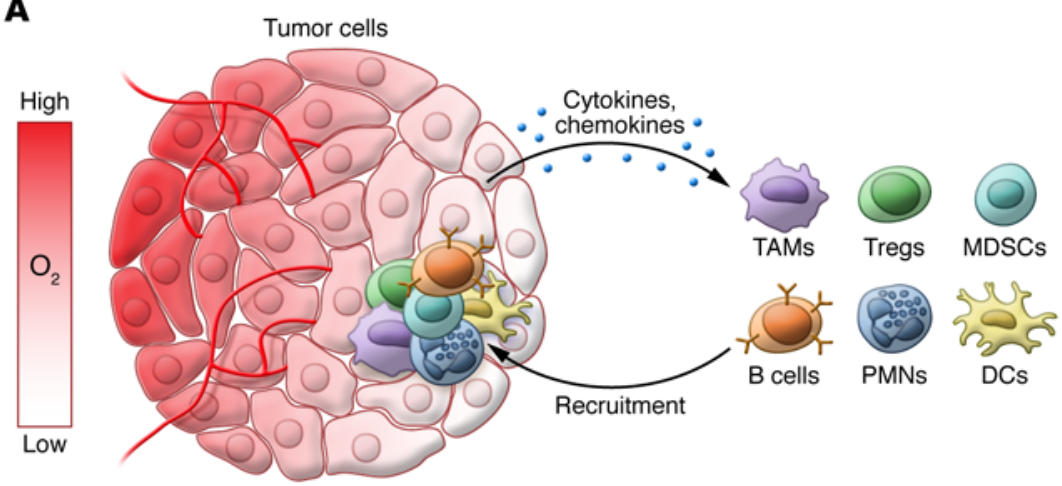

Figure 2. Hypoxic activation of tumor-promoting inflammatory responses. (A) As $\mathrm{O}_{2}$ concentration declines in tumors, hypoxia-directed secretion of cytokines and chemokines such as CXCL5, CXCL12, CCL28, and MIF recruits protumor immune cells including Tregs, TAMs, neutrophils, B cells, and MDSCs. (B) Recruited immune cells reside in hypoxic areas of tumors and hypoxia and HIFs regulate inflammatory cell effector functions to promote tumor growth. This includes suppression of antitumor immune responses, ROS production, angiogenesis, invasion/metastasis, drug resistance, and direct cytokine expression.

B

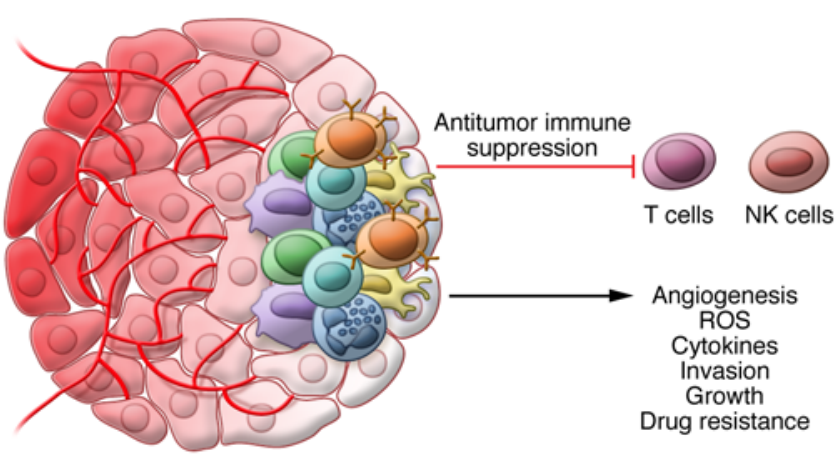

$T$ cells. $\mathrm{CD}^{+} \mathrm{T}$ cells can be differentiated into several different helper $\mathrm{T}(\mathrm{Th})$ cell types. Th1 and $\mathrm{Th} 2 \mathrm{CD}^{+}{ }^{+} \mathrm{T}$ cells are the classical types of Th cells that play an important role in the inflammatory response to infection and cancer (52). Th1 and Th2 cells promote antitumor immunity by cytotoxic lymphocyte activation and humoral-mediated immune responses, respectively (52). In addition to the classic Th1 and Th2 T cell effector populations, other subsets of $\mathrm{T}$ cells have been found to have an important role in cancer. For example, Th17 cells are a recently identified subset of IL-17-expressing $\mathrm{CD} 4^{+} \mathrm{T}$ cells that are highly prevalent in tumors and have a controversial role in tumor progression. IL- 6 and TGF- $\beta$ collaboratively promote Th17 differentiation (53) in a HIF-1 $\alpha$-dependent manner (54). Th17 cells have both anti- and protumor functions. In a melanoma model, Th17 cells showed more potent tumor eradication than Th1 cells (55). However, in other models, Th17 cells promote tumor growth through angiogenic and immunosuppressive effector functions $(56,57)$.

Immunosuppressive regulatory $\mathrm{T}$ cells (Tregs) are frequently increased in cancers (58). Tregs are $\mathrm{CD} 4{ }^{+}$and defined by expression of forkhead box transcription factor 3 (FoxP3). Tregs have been largely shown to promote tumorigenesis through suppression of antitumor $\mathrm{CD}^{+}$and $\mathrm{CD} 8^{+} \mathrm{T}$ cell-mediated immune responses by secreting immunosuppressive molecules such as IL-10 and TGF- $\beta$; removal of Tregs improves antitumor immunity (59-61). Primary tumor hypoxia regulated recruitment of $\mathrm{CCR} \mathrm{O}^{+} \mathrm{CD} 4^{+} \mathrm{FoxP}^{+}{ }^{+}$immunosuppressive Tregs in a model of ovarian cancer, which increased immune tolerance and angiogenesis through VEGF secretion (62). This effect was regulated by hypoxia-induced excretion of the chemokine CCL28, which was dependent upon HIF-1 $\alpha$ and to a lesser extent HIF-2 $\alpha$ (62).
$B$ cells. The precise role for B cells in tumor progression is controversial, and they may function to enhance or inhibit antitumor immune responses. B cells can be directly cytotoxic to tumors and promote antitumor $\mathrm{T}$ cell responses (63). However, B cells have also been shown to inhibit the function of antitumor $\mathrm{T}$ cells, as depletion of B cells increases antitumor immunity, suggesting context-specific roles for B cells in antitumor immunity (64). In pancreatic cancer, as detailed above, HIF-1 $\alpha$ in epithelial cells decreased tumor growth by attenuating expression of B cellrecruiting chemokines, resulting in decreased $\mathrm{B}$ cell infiltration into the tumors (46).

Myeloid-derived suppressor cells. Hypoxia also regulates tumor recruitment of immunosuppressive myeloid cells. Myeloid cells include monocytes/macrophages, neutrophils, eosinophils, basophils, mast cells, and dendritic cells (DCs). During infection or in cancers, immature myeloid cells that are closely related to neutrophils and monocytes can be detected in circulation. These cells, termed myeloid-derived suppressor cells (MDSCs), dampen immune responses to infection and promote tumor growth through suppression of both NK cell- and T cell-mediated antitumor immune responses (65). This tumor-promoting effect is mediated by multiple mechanisms including arginine metabolism via increased expression of arginase 1 (ARG1), which converts the available L-arginine pool to urea and L-ornithine. Additionally, MDSCs mediate nitration of tyrosine residues in the T cell receptor (TCR) and CD8, which decreases the function of these proteins (66-68). The function of MDSCs is well described in the azoxymethane (AOM) and dextran sulfate sodium (DSS) model of CAC. Mice with CXCR2-deficient bone marrow have significantly reduced MDSC homing to colon tumors and decreased colon tumorigenesis (69). Tumor-specific hypoxia increased recruitment of MDSCs 
Table 1. Regulation of immune cells by HIF-1 $\alpha$ and HIF-2 $\alpha$

\begin{tabular}{|c|c|c|}
\hline & \multicolumn{2}{|c|}{ Immune cell recruitment } \\
\hline & HIF-1 $1 \alpha$ & HIF- $2 \alpha$ \\
\hline TAM & $\begin{array}{l}\text { Promotes chemoattraction via SDF- } \\
1 \alpha / \text { CXCL12 expression (82) }\end{array}$ & Not known \\
\hline$D C$ & Not known & Not known \\
\hline PMN & Not known & $\begin{array}{l}\text { Represses CR-1+ } 1^{+} \text {granulocyte } \\
\text { recruitment to lung tumors ( } 43 \text { ) }\end{array}$ \\
\hline MDSC & $\begin{array}{l}\text { Increased recruitment to HNSCC } \\
\text { through MIF secretion (71) }\end{array}$ & $\begin{array}{l}\text { Increased recruitment to HNSCC } \\
\text { through MIF secretion (71) }\end{array}$ \\
\hline Treg & $\begin{array}{l}\text { Regulates CCL28 expression in ovarian } \\
\text { cancer (62) }\end{array}$ & Not known \\
\hline Th17 & Not known & Not known \\
\hline CD4 & Not known & Not known \\
\hline \multirow[t]{3}{*}{ B cell } & $\begin{array}{l}\text { Repress B cell infiltration into } \\
\text { pancreatic tumors (46) }\end{array}$ & Not known \\
\hline & Immune cell effector function & \\
\hline & HIF-1 $1 \alpha$ & HIF- $2 \alpha$ \\
\hline TAM & T cell suppression (96) & $\begin{array}{l}\text { Essential protumor function and } \\
\text { inflammatory responses (95) }\end{array}$ \\
\hline DC & Not known & Not known \\
\hline PMN & Decrease apoptosis (101) & $\begin{array}{l}\text { Regulate PMN-mediated } \\
\text { inflammatory responses (102) }\end{array}$ \\
\hline MDSC & $\begin{array}{l}\text { T cell suppression (98); Pdl1 } \\
\text { expression (100) }\end{array}$ & Not known \\
\hline Treg & $\begin{array}{l}\text { Regulates Foxp3 expression (110); } \\
\text { essential for immunosuppression }\end{array}$ & Not known \\
\hline Th17 & Essential for Th17 differentiation (54) & Not known \\
\hline CD4 & $\begin{array}{l}\text { Represses cytokine secretion (112); } \\
\text { regulates CD137 to augment antitumor } \\
\text { immunity (114) }\end{array}$ & Not known \\
\hline B cell & Essential for B cell maturation (144) & Not known \\
\hline
\end{tabular}

$\left(\mathrm{CD}_{11 \mathrm{~b}^{+}} \mathrm{Ly} 6 \mathrm{C}^{-}{ }^{-} \mathrm{Ly} 6 \mathrm{G}^{+}\right)$to the lung premetastatic niche to promote metastasis. This effect was partly regulated through inhibition of NK cell-mediated cytotoxicity (70). In head and neck squamous cell carcinoma, hypoxia increased recruitment of MDSCs in a process that was dependent upon both HIF-1 $\alpha$ and HIF-2 $\alpha$ induction of the potent chemoattractant migration inhibitory factor (MIF) (71).

Tumor-associated macrophages. The best-characterized immune cells in the tumor microenvironment are tumor-associated macrophages (TAMs). TAMs are highly prevalent in the tumor microenvironment and can be polarized into antitumor M1 or protumor/immunosuppressive M2 phenotypes (72). M2 TAMs regulate tumor angiogenesis and are an important source of VEGF (73). TAMs directly promote tumor growth via direct secretion of cytokines such as IL-6, which induces tumor-cell STAT3 signaling to promote growth and stem cell expansion (74); these cells have been directly linked to tumor invasion and metastasis $(75$, 76). TAMs inhibit antitumor immune responses through secretion of immunosuppressive cytokines such as IL-10 and TGF- $\beta$ (77). ARG1 expression in human monocytes and macrophages is controversial and has not been definitively shown in tumors (78). In rodents, however, there is clear evidence showing TAM expression of immunosuppressive ARG1 (79). TAMs reside in largely avascular and hypoxic regions of tumors (80). Tumor hypoxia is a potent driver of TAM recruitment and induces the secretion of chemoattractants such as oncostatin M and eotaxin (81). Moreover, tumor HIF-1 $\alpha$ directly induces recruitment of monocytes/ macrophages through regulation of stromal-derived factor $1 \alpha$ (SDF1a; also known as CXCL12) expression (82). Expression of the SDF1 $\alpha$ receptor CXCR4 is also regulated by hypoxia in TAMs (83). TAM polarization into a protumorigenic M2 phenotype can be directly regulated by tumor hypoxia. Tumor-derived lactic acid is induced by hypoxia in a HIF-1 $\alpha$-dependent manner and promotes M2 macrophage polarization and regulates expression of M2 TAM markers ARG1 and VEGF (84). Importantly, blockade of TAM recruitment to hypoxic tumor areas and trapping of TAMs in normoxic tumor microenvironments through loss of the semaphorin $3 \mathrm{~A}$ receptor NRP1 decreased tumor growth through blunted angiogenesis and increased antitumor $\mathrm{T}$ cell responses, showing that hypoxia-induced localization of macrophages causes a switch from anti- to protumor phenotypes (85).

Neutrophils. A close relationship between neutrophils (polymorphonuclear neutrophils [PMNs]) and tissue hypoxia has recently been shown. The reactive oxygen burst that is critical for neutrophil function can affect local tissue oxygenation (86). Although PMNs are highly prevalent in most solid tumor types, the specific role for PMNs is not completely understood. PMNs are highly plastic and can be differentiated into antitumor (N1) and protumor (N2) phenotypes (87). Protumor PMNs regulate tumor growth through secretion of cytokines, ROS production, generation of matrix-degrading enzymes, and angiogenesis (88). PMNs have also been shown to play an essential role in promoting metastasis in a murine breast cancer model (89). PMNs expressing the hepatocyte growth factor (HGF) receptor, c-MET, were found to be antitumorigenic in mouse models of colon cancer and liver cancer (90). PMNs have recently been demonstrated to reside in hypoxic tumor regions in epithelial uterine tumors and this effect was regulated by hypoxic tumor cell expression of PMN chemoattractants such as CXCL5 (91).

\section{Hypoxic regulation of tumor immune cell function}

Hypoxia is a hallmark of tumors and most infiltrating immune cells function in the hypoxic tumor environment. Immune cell expression of HIF-1 $\alpha$ and HIF-2 $\alpha$ regulates effector function (Table 1) (92). Tumor hypoxia has an essential role in regulating tumor inflammatory cell functions in addition to regulating immune cell recruitment (Figure 2B). TAMs express both HIF-1 $\alpha$ and HIF-2 $\alpha(16,93)$. TAM HIF-2 $\alpha$ expression is highly correlated with tumor vascularity and tumor grade (94). Macrophage HIF-2 $\alpha$ is critical in regulating macrophage inflammatory cytokine expression following LPS and IFN- $\gamma$ challenge (95). Importantly, macrophage loss of HIF-2 $\alpha$ impaired TAM infiltration of tumors and decreased tumor burden in murine models of HCC and CAC (95). TAM HIF-1 $\alpha$ has also been shown to play an important role in TAM-mediated suppression of tumor-associated T cells (96). TAMs cultured under hypoxic conditions exhibited increased suppression of T cells in a HIF-1 $\alpha$-dependent manner without affecting TAM recruitment or polarization (96). Furthermore, tumor hypoxia regulates TAM expression of VEGF, suggesting a role for hypoxic TAMs in angiogenesis (97). 
Hypoxia and HIF-1 $\alpha$ have an important role in regulating tumor-associated MDSC function. MDSCs cultured with the hypoxia mimetic desferroxamine (DFO) robustly suppress $\mathrm{T}$ cell proliferation and loss of HIF- $1 \alpha$ decreases MDSC-mediated T cell suppression (98). Hypoxia induces MDSC expression of miR-210 in a HIF-1 $\alpha$-dependent manner and miR-210 promotes MDSCmediated $\mathrm{T}$ cell suppression by increasing ARG1 expression and NO synthesis (99). Notably, HIF-1 $\alpha$ increases mRNA expression of the immune checkpoint receptor programmed death ligand-1 ( $P d l 1)$ in MDSCs, which is essential for their T cell immunosuppressive ability (100). MDSCs also have the capacity to differentiate into TAMs and HIF- $1 \alpha$ is a critical mediator of this plasticity (98).

The roles of PMN HIF- $1 \alpha$ and HIF- $2 \alpha$ in the tumor microenvironment are not well understood; however, it has been shown that PMN HIF- $1 \alpha$ is an essential PMN survival factor that mediates its effects through an NF- $\kappa \mathrm{B}$-dependent signaling loop (101). Constitutive HIF- $2 \alpha$ activation increases PMN inflammatory responses and loss of HIF-2 $\alpha$ increases susceptibility to apoptosis (102).

DCs are antigen-presenting cells (APCs) that are central regulators of the adaptive immune response. DCs can sample tumor antigens and activate $\mathrm{CD}^{+} \mathrm{T}$ cell responses and are currently in clinical trials as a vaccination strategy to prime antitumor immune responses (103). DC function is directly regulated by hypoxia and HIFs. Activated DCs increase expression of costimulatory molecules and $\mathrm{T}$ cell activation in response to TLR stimulation in a HIF- $1 \alpha-$ dependent manner when cultured under hypoxic conditions (104). Alternatively, it has been suggested that hypoxia-treated immature DCs have impaired antigen uptake and T cell activation $(105,106)$. Moreover, hypoxia inhibits DC maturation and $\mathrm{T}$ cell activation but simultaneously increases DC inflammatory cytokine secretion (107). Although the exact mechanisms are unclear, this dichotomy may be determined by the maturation state of DCs. Further work is needed to address more precisely the roles of DC HIF- $1 \alpha$ and HIF- $2 \alpha$ in the progression of cancer and antitumor immune responses.

Tumor hypoxia increases $\mathrm{T}$ cell expression of FoxP3 through tumor secretion of TGF- $\beta$ in vitro, suggesting that hypoxic tumor cells can induce Treg differentiation (108). Tregs are more efficiently activated systemically than within the tumor microenvironment (109), and tumor secretion of cytokines such as TGF- $\beta$ may be a critical source of systemic Treg activation and infiltration into tumors. CD $4^{+} \mathrm{T}$ cell HIF- $1 \alpha$ directly targets the Foxp3 promoter and increases immunosuppressive Treg cell production and function $(110,111)$. Loss of HIF- $1 \alpha$ in Tregs decreased their immunosuppressive function (110). The specific role for hypoxia signaling in antitumor $\mathrm{CD}^{+}$and $\mathrm{CD}^{+} \mathrm{T}$ cell responses is not completely clear. T cell HIF- $1 \alpha$ represses T cell inflammatory responses, as depletion of HIF- $1 \alpha$ significantly enhanced IFN- $\gamma$ and IL-2 production (112). However, other studies have shown that hypoxia signaling has the capacity to increase $\mathrm{CD} 8^{+} \mathrm{T}$ cell function, as $V h l$ depletion in $\mathrm{CD} 8^{+} \mathrm{T}$ cells increased $\mathrm{T}$ cell effector responses and decreased tumor growth in a model of melanoma (113). Additionally, HIF- $1 \alpha$ regulates expression of T cell CD137, which augments antitumor immunity upon antibody-mediated activation (114). More work is needed to better understand the specific roles of hypoxia and HIFs in antitumoral $\mathrm{T}$ cell responses. In addition, hypoxia has cell-intrinsic roles in CTLs, NK, and NKT cells, which have not been completely assessed in tumor biology.

\section{Hypoxia, stromal cells, and inflammation}

In addition to immune cells, the tumor microenvironment is also composed of vascular endothelial cells, fibroblasts, and pericytes, collectively known as the stroma. In tumor endothelial cells, depletion of HIF- $1 \alpha$ reduced tumor metastasis, whereas depletion of endothelial HIF- $2 \alpha$ increased tumor metastasis (115). This dichotomy was due to differential regulation of NO homeostasis by HIF- $1 \alpha$ and HIF- $2 \alpha$. HIF- $1 \alpha$ regulates expression of inducible nitric oxide synthase (iNOS), which catalyzes the conversion of L-arginine to NO. iNOS was also shown to be an essential regulator of VEGF expression and promoted increased tumor vascularity (115). Myeloid cell and mesenchymal stem cell NO production has been implicated in decreasing $\mathrm{T}$ cell cytotoxicity; thus, it is possible that endothelial cell NO has a similar function (116-118). On the other hand, HIF-2 $\alpha$ regulates expression of ARG1, which metabolizes L-arginine. ARG1 expression in immune cells is a potent suppressor of antitumor $\mathrm{T}$ cells. Future studies should determine if endothelial ARG1 also has an immunosuppressive role in the tumor microenvironment.

Fibroblasts are frequently recruited to tumors and make up a variable proportion of the tumor mass (119). Cancer-associated fibroblasts (CAFs) have a largely protumor role and promote angiogenesis through VEGF secretion and increase invasion/ metastasis through secretion of extracellular matrix-degrading matrix metalloproteinase enzymes (119-121). Conditional loss of CAF HIF-1 $\alpha$ by Fsp1-Cre expression decreased TAMs in mouse mammary tumors, suggesting that CAF HIF- $1 \alpha$ is important for TAM tumor infiltration (122). These results demonstrate that stromal cell HIF signaling can also modulate the microenvironment of tumors; however, the specific role for HIFs in the tumor endothelial cells and CAFs and the significance of this relationship to tumor inflammatory responses have not been fully elucidated.

\section{Hypoxia and intratumoral heterogeneity}

Tumor growth is an evolutionary process that leads to the accumulation of genetic alterations. This process also leads to substantial spatial variation within a tumor, which can contain genetically distinct subclonal populations of cancer cells (123). A few studies have demonstrated that intratumoral heterogeneity is an independent risk factor for poor survival in several tumor types, although further studies will be required to determine the effect of such heterogeneity in additional tumor types (124). The mechanisms that drive regional heterogeneity in tumors are poorly described. Hypoxia-induced immune cell recruitment and modulation could be important processes in driving regional selective pressures in tumors. More work is needed to elucidate the mechanisms by which hypoxia and immune cells establish microenvironments leading to intratumoral heterogeneity. Enhanced imaging techniques have made it clear that distinct subpopulations of hypoxic cells are present in tumors (125-127), while histopathological and molecular analyses have shown that distinct tumor regions have different inflammatory infiltrates that can be modulated by intratumoral hypoxia $(128,129)$.

\section{HIF-based therapeutics in cancer}

Due to the highly complex role of HIFs in tumorigenesis, HIFbased therapies need to be assessed on an individual-tumor basis. The best-characterized approach to alter HIF signaling is through 
PHD inhibition, which leads to activation of HIF signaling (130). PHD inhibitors have been assessed in models of inflammationinduced tissue injury and were shown to be effective and safe (131-133). Through a decrease in inflammation-induced tissue injury, PHD inhibitors may have a beneficial role in several cancers. Recent work has clearly demonstrated that PHD inhibitors can selectively activate HIF-1 $\alpha$ (134). This suggests the possibility of finding novel agents that may target each isoform. However, there are concerns about therapeutic activation of HIF signaling, as most data suggest that HIF pathway activation promotes tumor progression. Currently, there are several drugs that inhibit HIF-1 $\alpha$ and many of them are in clinical trials. Most of the compounds that are in clinical trials were originally discovered as targeting other pathways. 2-Methoxyestradiol, a metabolite of estradiol, can decrease tumor growth through inhibition of HIF-1a but simultaneously can decrease angiogenesis and disrupt microtubules (135). Cardiac glycosides, including digoxin, can robustly reduce tumor growth through inhibition of HIF-1 $\alpha$ (136). Furthermore, several topoisomerase inhibitors have been shown to decrease tumor growth through HIF-1 $\alpha$ inhibition $(137,138)$. However, no HIF-1 $\alpha$-specific inhibitors have been discovered. Selective inhibition of HIF- $2 \alpha$ can be achieved through targeting the iron response element in the $5^{\prime}$-UTR of HIF- $2 \alpha$ (139). Additionally, structural analysis of HIF- $2 \alpha$ identified a ligand-binding cavity located within the PAS-B domain, which contains a $\beta$-sheet that mediates interaction with ARNT; however, this region has not been identified on HIF-1 $\alpha$ (140). There are no known endogenous ligands for HIF-2 $\alpha$, but this cavity has been targeted for drug development and several promising highly specific smallmolecule inhibitors have been identified that efficaciously disrupt HIF- $2 \alpha$ heterodimerization with ARNT and block DNA binding and transcription of target genes in cultured cells (141). These reagents coupled with recent HIF structural analysis provide a framework to specifically regulate HIF-1 $\alpha$ and HIF- $2 \alpha$ and will aid the development of clinical tools to alter protumor inflammatory responses or antitumor immune responses (142).

\section{Conclusion}

Hypoxia is an important microenvironmental feature in solid tumors and is essential for tumor growth. HIF- $1 \alpha$ and HIF- $2 \alpha$ have been extensively studied in regulating tumor glucose metabolism, angiogenesis, cell survival, proliferation, and migration. Research in the past two decades has established an essential role for protumor inflammatory responses or antitumor immune responses in the growth of most solid tumors and with this increased focus, Hanahan and Weinberg's "hallmarks of cancer" were recently updated to include tumor inflammation and immune evasion as a major enabling factor in cancer progression (143). It is becoming clear that hypoxia is central to regulating the inflammatory response in tumors. Future studies should be directed towards a better understanding of the precise molecular mechanisms by which hypoxia alters the balance between growth-promoting inflammation and the antitumor immune response, which may lead to better use of existing drugs that alter the HIF response as stand-alone therapies and improve the efficacy of standard chemotherapeutics.

\section{Acknowledgments}

This work was supported by NIH grants CA148828 and DK095201 (to Y.M. Shah), and D. Triner was funded by the T32 training grant (T32 DK 094775) "Training in Basic and Translational Digestive Sciences."

Address correspondence to: Yatrik M. Shah, Department of Molecular and Integrative Physiology, Department of Internal medicine, Division of Gastroenterology, University of Michigan Medical School, Ann Arbor, Michigan 48109, USA. Phone: 734.615.0567; E-mail:shahy@umich.edu.
1. Gajewski TF, Schreiber H, Fu YX. Innate and adaptive immune cells in the tumor microenvironment. Nat Immunol. 2013;14(10):1014-1022.

2. Semenza GL. Hypoxia-inducible factors in physiology and medicine. Cell. 2012;148(3):399-408.

3. Wiesener MS, et al. Widespread hypoxiainducible expression of HIF-2alpha in distinct cell populations of different organs. FASEB J. 2003;17(2):271-273.

4. Wiener CM, Booth G, Semenza GL. In vivo expression of mRNAs encoding hypoxiainducible factor 1. Biochem Biophys Res Commun. 1996;225(2):485-488.

5. Gu YZ, Moran SM, Hogenesch JB, Wartman L, Bradfield CA. Molecular characterization and chromosomal localization of a third alpha-class hypoxia inducible factor subunit, HIF3alpha. Gene Expr. 1998;7(3):205-213.

6. Rius J, et al. NF-kappaB links innate immunity to the hypoxic response through transcriptional regulation of HIF-1alpha. Nature. 2008;453(7196):807-811.

7. Siegert I, et al. ferritin-mediated iron sequestration stabilizes hypoxia-inducible factor- $1 \alpha$ upon LPS activation in the presence of ample oxygen.
Cell Rep. 2015;13(10):2048-2055.

8. Haddad JJ, Harb HL. Cytokines and the regulation of hypoxia-inducible factor (HIF)-1alpha. Int Immunopharmacol. 2005;5(3):461-483.

9. Tannahill GM, et al. Succinate is an inflammatory signal that induces IL-1 $\beta$ through HIF- $1 \alpha$. Nature. 2013;496(7444):238-242.

10. Takeda N, et al. Differential activation and antagonistic function of HIF- $\alpha$ isoforms in macrophages are essential for NO homeostasis. Genes Dev. 2010;24(5):491-501.

11. Hellwig-Bürgel T, Rutkowski K, Metzen E, Fandrey J, Jelkmann W. Interleukin-1beta and tumor necrosis factor-alpha stimulate DNA binding of hypoxia-inducible factor-1. Blood. 1999;94(5):1561-1567.

12. Haddad JJ, Land SC. A non-hypoxic, ROSsensitive pathway mediates TNF-alphadependent regulation of HIF-1alpha. FEBS Lett. 2001;505(2):269-274.

13. Chandel NS, Maltepe E, Goldwasser E, Mathieu CE, Simon MC, Schumacker PT. Mitochondrial reactive oxygen species trigger hypoxiainduced transcription. Proc Natl Acad Sci U S A. 1998;95(20):11715-11720.
14. Martínez-Reyes I, et al. TCA cycle and mitochondrial membrane potential are necessary for diverse biological functions. Mol Cell. 2016;61(2):199-209.

15. Semenza GL. Hypoxia-inducible factors: mediators of cancer progression and targets for cancer therapy. Trends Pharmacol Sci. 2012;33(4):207-214.

16. Talks KL, et al. The expression and distribution of the hypoxia-inducible factors HIF-1alpha and HIF-2alpha in normal human tissues, cancers, and tumor-associated macrophages. Am J Pathol. 2000;157(2):411-421.

17. Hu CJ, Wang LY, Chodosh LA, Keith B, Simon MC. Differential roles of hypoxia-inducible factor 1alpha (HIF-1alpha) and HIF-2alpha in hypoxic gene regulation. Mol Cell Biol. 2003;23(24):9361-9374.

18. Keith B, Johnson RS, Simon MC. HIF1 $\alpha$ and HIF2 $\alpha$ : sibling rivalry in hypoxic tumour growth and progression. Nat Rev Cancer. 2012;12(1):9-22.

19. Raval RR, et al. Contrasting properties of hypoxia-inducible factor 1 (HIF-1) and HIF-2 in von Hippel-Lindau-associated renal cell carcinoma. Mol Cell Biol. 2005;25(13):5675-5686.

20. Gordan JD, Bertout JA, Hu CJ, Diehl JA, Simon 
MC. HIF-2alpha promotes hypoxic cell proliferation by enhancing c-myc transcriptional activity. Cancer Cell. 2007;11(4):335-347.

21. Palazon A, Goldrath AW, Nizet V, Johnson RS. HIF transcription factors, inflammation, and immunity. Immunity. 2014;41(4):518-528.

22. Pardoll DM. The blockade of immune checkpoints in cancer immunotherapy. Nat Rev Cancer. 2012;12(4):252-264.

23. Ahn HJ, Lee DS. Helicobacter pylori in gastric carcinogenesis. World J Gastrointest Oncol. 2015;7(12):455-465.

24. Mitra AK. Hepatitis C-related hepatocellular carcinoma: prevalence around the world, factors interacting, and role of genotypes. Epidemiol Rev. 1999;21(2):180-187.

25. Munkholm P. Review article: the incidence and prevalence of colorectal cancer in inflammatory bowel disease. Aliment Pharmacol Ther. 2003;18 Suppl 2:1-5.

26. Fearon ER. Molecular genetics of colorectal cancer. Annu Rev Pathol. 2011;6:479-507.

27. Fearon ER, Vogelstein B. A genetic model for colorectal tumorigenesis. Cell. 1990;61(5):759-767.

28. Kim ER, Chang DK. Colorectal cancer in inflammatory bowel disease: the risk, pathogenesis, prevention and diagnosis. World J Gastroenterol. 2014;20(29):9872-9881.

29. Robles AI, et al. Whole-exome sequencing analyses of inflammatory bowel diseaseassociated colorectal cancers. Gastroenterology. 2016;150(4):931-943.

30. Grivennikov SI, Greten FR, Karin M. Immunity, inflammation, and cancer. Cell. 2010;140(6):883-899.

31. Schwitalla S, et al. Intestinal tumorigenesis initiated by dedifferentiation and acquisition of stemcell-like properties. Cell. 2013;152(1-2):25-38.

32. Sparmann A, Bar-Sagi D. Ras-induced interleukin-8 expression plays a critical role in tumor growth and angiogenesis. Cancer Cell. 2004;6(5):447-458.

33. Grivennikov SI, et al. Adenoma-linked barrier defects and microbial products drive IL-23/IL-17-mediated tumour growth. Nature. 2012;491(7423):254-258.

34. Galon J, et al. Type, density, and location of immune cells within human colorectal tumors predict clinical outcome. Science. 2006;313(5795):1960-1964.

35. Gentles AJ, et al. The prognostic landscape of genes and infiltrating immune cells across human cancers. Nat Med. 2015;21(8):938-945.

36. Sato $\mathrm{E}$, et al. Intraepithelial $\mathrm{CD} 8^{+}$tumor-infiltrating lymphocytes and a high $\mathrm{CD} 8^{+} /$regulatory $\mathrm{T}$ cell ratio are associated with favorable prognosis in ovarian cancer. Proc Natl Acad Sci U S A. 2005;102(51):18538-18543.

37. Pagès $\mathrm{F}$, et al. Effector memory $\mathrm{T}$ cells, early metastasis, and survival in colorectal cancer. N Engl J Med. 2005;353(25):2654-2666.

38. Shaykhiev R, Bals R. Interactions between epithelial cells and leukocytes in immunity and tissue homeostasis. J Leukoc Biol. 2007;82(1):1-15.

39. Artis D. Epithelial-cell recognition of commensal bacteria and maintenance of immune homeostasis in the gut. Nat Rev Immunol. 2008;8(6):411-420.

40. Chun E, et al. CCL2 promotes colorectal carcinogenesis by enhancing polymorphonuclear myeloid-derived suppressor cell population and function. Cell Rep. 2015;12(2):244-257.

41. Bonavita E, et al. PTX3 is an extrinsic oncosuppressor regulating complement-dependent inflammation in cancer. Cell. 2015;160(4):700-714.

42. Isella $\mathrm{C}$, et al. Stromal contribution to the colorectal cancer transcriptome. Nat Genet. 2015;47(4):312-319.

43. Mazumdar J, et al. HIF-2alpha deletion promotes Kras-driven lung tumor development. Proc Natl Acad Sci U S A. 2010;107(32):14182-14187.

44. Vincent A, Herman J, Schulick R, Hruban RH, Goggins M. Pancreatic cancer. Lancet. 2011;378(9791):607-620.

45. Criscimanna A, et al. PanIN-specific regulation of Wnt signaling by HIF2 $\alpha$ during early pancreatic tumorigenesis. Cancer Res. 2013;73(15):4781-4790.

46. Lee KE, et al. Hifla deletion reveals pro-neoplastic function of $\mathrm{B}$ cells in pancreatic neoplasia. Cancer Discov. 2016;6(3):256-269.

47. Xue X, et al. Hypoxia-inducible factor- $2 \alpha$ activation promotes colorectal cancer progression by dysregulating iron homeostasis. Cancer Res. 2012;72(9):2285-2293.

48. Xue X, et al. Endothelial PAS domain protein 1 activates the inflammatory response in the intestinal epithelium to promote colitis in mice. Gastroenterology. 2013;145(4):831-841.

49. Popivanova BK, et al. Blocking TNF-alpha in mice reduces colorectal carcinogenesis associated with chronic colitis. JClin Invest. 2008;118(2):560-570.

50. Zhao X, et al. TNF signaling drives myeloidderived suppressor cell accumulation. JClin Invest. 2012;122(11):4094-4104.

51. Xue X, Ramakrishnan SK, Shah YM. Activation of HIF-1 $\alpha$ does not increase intestinal tumorigenesis. Am J Physiol Gastrointest Liver Physiol. 2014;307(2):G187-G195.

52. Knutson KL, Disis ML. Tumor antigen-specific $T$ helper cells in cancer immunity and immunotherapy. Cancer Immunol Immunother. 2005;54(8):721-728.

53. Bettelli E, et al. Reciprocal developmental pathways for the generation of pathogenic effector TH17 and regulatory T cells. Nature. 2006;441(7090):235-238.

54. Dang EV, et al. Control of T(H)17/T(reg) balance by hypoxia-inducible factor 1. Cell. 2011;146(5):772-784.

55. Muranski P, et al. Tumor-specific Th17-polarized cells eradicate large established melanoma. Blood. 2008;112(2):362-373.

56. Wang L, Yi T, Zhang W, Pardoll DM, Yu H. IL-17 enhances tumor development in carcinogen-induced skin cancer. Cancer Res. 2010;70(24):10112-10120.

57. Chang SH, et al. T helper 17 cells play a critical pathogenic role in lung cancer. Proc Natl Acad Sci U S A. 2014;111(15):5664-5669.

58. Adeegbe DO, Nishikawa H. Natural and induced T regulatory cells in cancer. Front Immunol. 2013;4:190

59. Li X, Kostareli E, Suffner J, Garbi N, Hämmerling GJ. Efficient Treg depletion induces T-cell infiltration and rejection of large tumors. Eur $J$ Immunol.2010;40(12):3325-3335.

60. Klages K, et al. Selective depletion of Foxp $3^{+}$ regulatory T cells improves effective therapeutic vaccination against established melanoma. Cancer Res. 2010;70(20):7788-7799.

61. Sakaguchi S, Wing K, Onishi Y, Prieto-Martin P, Yamaguchi T. Regulatory T cells: how do they suppress immune responses? Int Immunol. 2009;21(10):1105-1111.

62. Facciabene A, et al. Tumour hypoxia promotes tolerance and angiogenesis via CCL28 and T(reg) cells. Nature. 2011;475(7355):226-230.

63. $\mathrm{Li} \mathrm{Q}$, et al. Adoptive transfer of tumor reactive $\mathrm{B}$ cells confers host T-cell immunity and tumor regression. Clin Cancer Res. 2011;17(15):4987-4995.

64. Kim S, et al. B-cell depletion using an anti-CD2O antibody augments antitumor immune responses and immunotherapy in nonhematopoetic murine tumor models. JImmunother. 2008;31(5):446-457.

65. Gabrilovich DI, Nagaraj S. Myeloid-derived suppressor cells as regulators of the immune system. Nat Rev Immunol. 2009;9(3):162-174.

66. Lu T, et al. Tumor-infiltrating myeloid cells induce tumor cell resistance to cytotoxic $\mathrm{T}$ cells in mice. J Clin Invest. 2011;121(10):4015-4029.

67. Rodríguez PC, Ochoa AC. Arginine regulation by myeloid derived suppressor cells and tolerance in cancer: mechanisms and therapeutic perspectives. Immunol Rev. 2008;222:180-191.

68. Nagaraj $\mathrm{S}$, et al. Altered recognition of antigen is a mechanism of $\mathrm{CD} 8^{+} \mathrm{T}$ cell tolerance in cancer. Nat Med. 2007;13(7): 828-835.

69. Katoh H, Wang D, Daikoku T, Sun H, Dey SK, Dubois RN. CXCR2-expressing myeloidderived suppressor cells are essential to promote colitis-associated tumorigenesis. Cancer Cell. 2013;24(5):631-644.

70. Sceneay J, et al. Primary tumor hypoxia recruits $\mathrm{CD}_{11 \mathrm{~b}^{+} / \mathrm{Ly} 6 \mathrm{C}^{\text {med }} / \mathrm{Ly} 6 \mathrm{G}^{+} \text {immune suppressor }}$ cells and compromises NK cell cytotoxicity in the premetastatic niche. Cancer Res. 2012;72(16):3906-3911.

71. Zhu G, et al. HIF- $\alpha /$ MIF and NF- $\mathrm{BB} / \mathrm{IL}-6$ axes contribute to the recruitment of $\mathrm{CD} 11 \mathrm{~b}^{+} \mathrm{Gr}-\mathrm{1}^{+}$ myeloid cells in hypoxic microenvironment of HNSCC. Neoplasia. 2014;16(2):168-179.

72. Sica A, Mantovani A. Macrophage plasticity and polarization: in vivo veritas. JClin Invest. 2012;122(3):787-795.

73. Lin EY, et al. Macrophages regulate the angiogenic switch in a mouse model of breast cancer. Cancer Res. 2006;66(23):11238-11246.

74. Wan S, et al. Tumor-associated macrophages produce interleukin 6 and signal via STAT3 to promote expansion of human hepatocellular carcinoma stem cells. Gastroenterology. 2014;147(6):1393-1404.

75. Hagemann T, et al. Macrophages induce invasiveness of epithelial cancer cells via NF-kappa B and JNK. J Immunol. 2005;175(2):1197-1205.

76. Gocheva V, et al. IL-4 induces cathepsin protease activity in tumor-associated macrophages to promote cancer growth and invasion. Genes Dev. 2010;24(3):241-255.

77. Hao NB, Lü MH, Fan YH, Cao YL, Zhang ZR, Yang SM. Macrophages in tumor microenvironments and the progression of tumors. Clin Dev Immunol. 2012;2012:948098.

78. Thomas AC, Mattila JT. "Of mice and men": arginine metabolism in macrophages. Front Immunol. 
2014;5:479.

79. Rodriguez PC, et al. Arginase I production in the tumor microenvironment by mature myeloid cells inhibits T-cell receptor expression and antigen-specific T-cell responses. Cancer Res. 2004;64(16):5839-5849.

80. Lewis C, Murdoch C. Macrophage responses to hypoxia: implications for tumor progression and anti-cancer therapies. Am J Pathol. 2005;167(3):627-635.

81. Tripathi $\mathrm{C}$, et al. Macrophages are recruited to hypoxic tumor areas and acquire a pro-angiogenic M2-polarized phenotype via hypoxic cancer cell derived cytokines Oncostatin $\mathrm{M}$ and Eotaxin. Oncotarget. 2014;5(14):5350-5368.

82. Du R, et al. HIF1alpha induces the recruitment of bone marrow-derived vascular modulatory cells to regulate tumor angiogenesis and invasion. Cancer Cell. 2008;13(3):206-220.

83. Schioppa T, et al. Regulation of the chemokine receptor CXCR 4 by hypoxia. JExp Med. 2003;198(9):1391-1402.

84. Colegio OR, et al. Functional polarization of tumour-associated macrophages by tumour-derived lactic acid. Nature. 2014;513(7519):559-563.

85. Casazza A, et al. Impeding macrophage entry into hypoxic tumor areas by Sema3A/Nrp1 signaling blockade inhibits angiogenesis and restores antitumor immunity. Cancer Cell. 2013;24(6):695-709.

86. Campbell EL, et al. Transmigrating neutrophils shape the mucosal microenvironment through localized oxygen depletion to influence resolution of inflammation. Immunity. 2014;40(1):66-77.

87. Fridlender ZG, et al. Polarization of tumor-associated neutrophil phenotype by TGF-beta: "N1" versus “N2" TAN. Cancer Cell. 2009;16(3):183-194.

88. Galdiero MR, Bonavita E, Barajon I, Garlanda C, Mantovani A, Jaillon S. Tumor associated macrophages and neutrophils in cancer. Immunobiology. 2013;218(11):1402-1410.

89. Coffelt SB, et al. IL-17-producing $\gamma \delta$ T cells and neutrophils conspire to promote breast cancer metastasis. Nature. 2015;522(7556):345-348.

90. Finisguerra $V$, et al. MET is required for the recruitment of anti-tumoural neutrophils. Nature. 2015;522(7556):349-353.

91. Blaisdell A, et al. Neutrophils oppose uterine epithelial carcinogenesis via debridement of hypoxic tumor cells. Cancer Cell. 2015;28(6):785-799.

92. Cummins EP, Keogh CE, Crean D, Taylor CT. The role of HIF in immunity and inflammation. Mol Aspects Med. 2016;47-48:24-34.

93. Burke B, et al. Expression of HIF-1alpha by human macrophages: implications for the use of macrophages in hypoxia-regulated cancer gene therapy. J Pathol. 2002;196(2):204-212.

94. Leek RD, et al. Relation of hypoxia-inducible factor-2 $\alpha$ (HIF-2 $\alpha$ ) expression in tumor-infiltrative macrophages to tumor angiogenesis and the oxidative thymidine phosphorylase pathway in human breast cancer. Cancer Res. 2002;62(5):1326-1329.

95. Imtiyaz HZ, et al. Hypoxia-inducible factor 2alpha regulates macrophage function in mouse models of acute and tumor inflammation. JClin Invest. 2010;120(8):2699-2714.

96. Doedens AL, et al. Macrophage expression of hypoxia-inducible factor-1 alpha suppresses $\mathrm{T}$-cell function and promotes tumor progression. Cancer Res. 2010;70(19):7465-7475.

97. Riabov V, Gudima A, Wang N, Mickley A, Orekhov A, Kzhyshkowska J. Role of tumor associated macrophages in tumor angiogenesis and lymphangiogenesis. Front Physiol. 2014;5:75.

98. Corzo CA, et al. HIF-1 $\alpha$ regulates function and differentiation of myeloid-derived suppressor cells in the tumor microenvironment. JExp Med. 2010;207(11):2439-2453.

99. Noman MZ, et al. Tumor-promoting effects of myeloid-derived suppressor cells are potentiated by hypoxia-induced expression of miR-210. Cancer Res. 2015;75(18):3771-3787.

100.Noman MZ, et al. PD-L1 is a novel direct target of HIF-1 $\alpha$, and its blockade under hypoxia enhanced MDSC-mediated T cell activation. J Exp Med. 2014;211(5):781-790.

101. Walmsley SR, et al. Hypoxia-induced neutrophil survival is mediated by HIF-1alpha-dependent NFkappaB activity. JExp Med. 2005;201(1):105-115

102. Thompson AA, et al. Hypoxia-inducible factor $2 \alpha$ regulates key neutrophil functions in humans, mice, and zebrafish. Blood. 2014;123(3):366-376.

103. Palucka K, Banchereau J. Cancer immunotherapy via dendritic cells. Nat Rev Cancer. 2012;12(4):265-277.

104.Jantsch J, et al. Hypoxia and hypoxia-inducible factor-1 alpha modulate lipopolysaccharideinduced dendritic cell activation and function. JImmunol. 2008;180(7):4697-4705.

105. Oppenheim JJ, Rosenberg HF. The 2008 Dolph Adams award and the state of the journal of leukocyte biology. J Leukoc Biol. 2008;84(6):1361-1362.

106. Yang M, et al. Hypoxia skews dendritic cells to a Thelper type 2-stimulating phenotype and promotes tumour cell migration by dendritic cellderived osteopontin. Immunology. 2009; 128(1 Suppl):e237-e249.

107. Mancino A, et al. Divergent effects of hypoxia on dendritic cell functions. Blood. 2008;112(9):3723-3734.

108. Deng B, et al. Intratumor hypoxia promotes immune tolerance by inducing regulatory $\mathrm{T}$ cells via TGF- $\beta 1$ in gastric cancer. PLOS ONE. 2013;8(5):e63777.

109. Wang C, Lee JH, Kim CH. Optimal population of $\mathrm{FoxP}^{+} \mathrm{T}$ cells in tumors requires an antigen priming-dependent trafficking receptor switch. PLoS ONE. 2012;7(1):e30793.

110.Clambey ET, et al. Hypoxia-inducible factor-1 alpha-dependent induction of FoxP3 drives regulatory T-cell abundance and function during inflammatory hypoxia of the mucosa. Proc Natl Acad Sci U S A. 2012;109(41):E2784-E2793.

111. Ben-Shoshan J, Maysel-Auslender S, Mor A, Keren G, George J. Hypoxia controls $\mathrm{CD} 4{ }^{+} \mathrm{CD} 25^{+}$regulatory T-cell homeostasis via hypoxia-inducible factor-1alpha. Eur J Immunol. 2008;38(9):2412-2418.

112. Lukashev D, et al. Cutting edge: hypoxia-inducible factor 1alpha and its activation-inducible short isoform I.1 negatively regulate functions of $\mathrm{CD}^{+}$and $\mathrm{CD} 8^{+} \mathrm{T}$ lymphocytes. Jimmunol. 2006;177(8):4962-4965.

113. Doedens AL, et al. Hypoxia-inducible factors enhance the effector responses of $\mathrm{CD} 8(+)$
T cells to persistent antigen. Nat Immunol. 2013;14(11):1173-1182.

114. Palazón A, et al. The HIF-1 $\alpha$ hypoxia response in tumor-infiltrating T lymphocytes induces functional CD137 (4-1BB) for immunotherapy. Cancer Discov. 2012;2(7):608-623.

115. Branco-Price C, et al. Endothelial cell HIF-1 $\alpha$ and HIF-2 $\alpha$ differentially regulate metastatic success. Cancer Cell. 2012;21(1):52-65.

116. Mazzoni A, et al. Myeloid suppressor lines inhibit $\mathrm{T}$ cell responses by an NO-dependent mechanism. J Immunol. 2002;168(2):689-695.

117. Medot-Pirenne M, Heilman MJ, Saxena M, McDermott PE, Mills CD. Augmentation of an antitumor CTL response in vivo by inhibition of suppressor macrophage nitric oxide. JImmunol. 1999;163(11):5877-5882.

118. Sato K, et al. Nitric oxide plays a critical role in suppression of T-cell proliferation by mesenchymal stem cells. Blood. 2007;109(1):228-234.

119. Kalluri R, Zeisberg M. Fibroblasts in cancer. Nat Rev Cancer. 2006;6(5):392-401.

120.Boire A, Covic L, Agarwal A, Jacques S, Sherifi S, Kuliopulos A. PAR1 is a matrix metalloprotease-1 receptor that promotes invasion and tumorigenesis of breast cancer cells. Cell. 2005;120(3):303-313.

121. Hlatky L, Tsionou C, Hahnfeldt P, Coleman $\mathrm{CN}$. Mammary fibroblasts may influence breast tumor angiogenesis via hypoxia-induced vascular endothelial growth factor up-regulation and protein expression. Cancer Res. 1994;54(23):6083-6086.

122. Kim JW, et al. Loss of fibroblast HIF-1a accelerates tumorigenesis. Cancer Res. 2012;72(13):3187-3195.

123. McGranahan N, Swanton C. Biological and therapeutic impact of intratumor heterogeneity in cancer evolution. Cancer Cell. 2015;27(1):15-26.

124. Yachida S, et al. Distant metastasis occurs late during the genetic evolution of pancreatic cancer. Nature. 2010;467(7319):1114-1117.

125. Cui YL, Wang X, Li XF. ${ }^{18} \mathrm{~F}$-fluoromisonidazole $P E T$ reveals spatial and temporal heterogeneity of hypoxia in mouse models of human non-small-cell lung cancer. Future Oncol. 2015;11(20):2841-2849.

126.Zornhagen KW, et al. Micro regional heterogeneity of ${ }^{64} \mathrm{Cu}$-ATSM and ${ }^{18} \mathrm{~F}$-FDG uptake in canine soft tissue sarcomas: relation to cell proliferation, hypoxia and glycolysis. PLOS ONE. 2015;10(10):e0141379.

127. Erapaneedi R, Belousov VV, Schäfers M, Kiefer F. A novel family of fluorescent hypoxia sensors reveal strong heterogeneity in tumor hypoxia at the cellular level. EMBO J. 2016;35(1):102-113.

128. Movahedi K, et al. Different tumor microenvironments contain functionally distinct subsets of macrophages derived from Ly6C(high) monocytes. Cancer Res. 2010;70(14):5728-5739.

129. Laoui D, et al. Tumor hypoxia does not drive differentiation of tumor-associated macrophages but rather fine-tunes the M2-like macrophage population. Cancer Res. 2014;74(1):24-30.

130. Rabinowitz MH. Inhibition of hypoxia-inducible factor prolyl hydroxylase domain oxygen sensors: tricking the body into mounting orchestrated survival and repair responses. JMed Chem. 2013;56(23):9369-9402. 
131. Robinson A, Keely S, Karhausen J, Gerich ME, Furuta GT, Colgan SP. Mucosal protection by hypoxia-inducible factor prolyl hydroxylase inhibition. Gastroenterology. 2008;134(1):145-155.

132. Cummins EP, et al. The hydroxylase inhibitor dimethyloxalylglycine is protective in a murine model of colitis. Gastroenterology. 2008;134(1):156-165.

133. Eckle T, et al. HIF1A reduces acute lung injury by optimizing carbohydrate metabolism in the alveolar epithelium. PLoS Biol. 2013;11(9):e1001665.

134. Keely S, et al. Contribution of epithelial innate immunity to systemic protection afforded by prolyl hydroxylase inhibition in murine colitis. Mucosal Immunol. 2014;7(1):114-123.

135. Mabjeesh NJ, et al. 2ME2 inhibits tumor growth and angiogenesis by disrupting microtubules and dysregulating HIF. Cancer Cell. 2003;3(4):363-375.
136.Zhang $\mathrm{H}$, et al. Digoxin and other cardiac glycosides inhibit HIF-1alpha synthesis and block tumor growth. Proc Natl Acad Sci U S A. 2008;105(50):19579-19586.

137. Beppu K, Nakamura K, Linehan WM, Rapisarda A, Thiele CJ. Topotecan blocks hypoxia-inducible factor-1alpha and vascular endothelial growth factor expression induced by insulin-like growth factor-I in neuroblastoma cells. Cancer Res. 2005;65(11):4775-4781.

138. Pencreach E, et al. Marked activity of irinotecan and rapamycin combination toward colon cancer cells in vivo and in vitro is mediated through cooperative modulation of the mammalian target of rapamycin/hypoxia-inducible factor-1alpha axis. Clin Cancer Res. 2009;15(4):1297-1307.

139. Zimmer M, et al. Small-molecule inhibitors of HIF-2a translation link its 5'UTR ironresponsive element to oxygen sensing. Mol Cell.
2008;32(6):838-848.

140.Scheuermann TH, Tomchick DR, Machius M, Guo Y, Bruick RK, Gardner KH. Artificial ligand binding within the HIF2alpha PAS-B domain of the HIF2 transcription factor. Proc Natl Acad Sci US A. 2009;106(2):450-455.

141. Scheuermann TH, et al. Allosteric inhibition of hypoxia inducible factor- 2 with small molecules. Nat Chem Biol. 2013;9(4):271-276.

142.Wu D, Potluri N, Lu J, Kim Y, Rastinejad F. Structural integration in hypoxia-inducible factors. Nature. 2015;524(7565):303-308.

143. Hanahan D, Weinberg RA. Hallmarks of cancer: the next generation. Cell. 2011;144(5):646-674.

144.Kojima H, et al. Differentiation stage-specific requirement in hypoxia-inducible factor-1alpharegulated glycolytic pathway during murine $\mathrm{B}$ cell development in bone marrow. JImmunol. 2010;184(1):154-163. 